\title{
Weighted Alignment Measures of Enterprise Architecture Viewpoints
}

\author{
Jacques Simonin $^{1}$, Selmin Nurcan ${ }^{2}$, and Julie Gourmelen ${ }^{3}$ \\ ${ }^{1}$ Institut Mines-Télécom / Télécom Bretagne, Lab-STICC UMR CNRS 6285, UEB, \\ Technopôle Brest-Iroise, 29238 Brest, France \\ jacques.simoninatelecom-bretagne.eu \\ ${ }^{2}$ Sorbonne Business School, University Paris 1 Panthéon Sorbonne, \\ 90 rue de Tolbiac, 75013 Paris, France \\ selmin. nurcan@univ-paris1. fr \\ ${ }^{3}$ INSERM, U1018, Université Versailles Saint Quentin, Hôpital Paul Brousse, \\ 16 avenue Vaillant-Couturier, 94800, Villejuif, France \\ julie.gourmelen@inserm. fr
}

\begin{abstract}
Enterprise Architecture (EA) allows describing how an organization can achieve its objectives and/or develop innovative strategies, through the creation of a set of engineered models that can be understood by the people associated with the organization. To this end, the set of EA models should be developed as any product in any engineering domain. In general, those models are the artifacts on which we reason to understand the alignment between several viewpoints of the organization. Reasoning on those artefacts in an objective and systematic way requires a shared way to represent them and a set of weighted measures based on this way-of-representing. This paper proposes a framework to analyze and to represent multiple viewpoints of an organization and develops a set of measures to qualify the alignment between those models. These measures are experimented from the alignment of Information System functions with respect to business processes for medical research.
\end{abstract}

Keywords: Enterprise Architecture, Viewpoint, Model, Systemic, Alignment, Measure, Weight.

\section{Introduction}

\subsection{Context and Motivation}

Enterprise Architecture (EA) [1] frameworks enable to represent and to analyze multiple viewpoints of an organization. They define an organization system based on those viewpoints. Modeling capabilities are needed to perform this engineering activity and each viewpoint owns its specific set of concepts to this end. In this context, model alignment [2] remains a prerequisite for the organization viewpoints stakeholders who design the organization system using several viewpoint models [3]. A viewpoint describes a partitioning of concepts enabling a modeling of a given system of the organization in relation to this viewpoint [4]. Moreover, thinking in terms of viewpoints allows dividing the system into specific areas of expertise [5]. 
The EA viewpoints alignment definition does not depend on the EA framework choice. We consider in this paper that the alignment between EA viewpoints may be approached from the alignment of models representing each of these viewpoints.

Nevertheless, many problems may prejudice the alignment estimation. In fact, viewpoint models in organization are of varying quality or simply inexistent. Our hypothesis in this work is that those models exist and they conform to viewpoints meta-modeling. The alignment is thus defined from this meta-model and applied to viewpoint models.

This paper proposes two new model alignment measures meaning EA viewpoint model concept estimation and viewpoint model global estimation. This estimation is based on a set of quantified measures to score the alignment between the viewpoint models in terms of ratios (values inside the interval $[0,1]$ ).

We propose in Section 2 an alignment definition of viewpoint models. In Section 3 , the viewpoint models alignment is then evaluated from weighted alignment measures. An epidemiological IS of the French National Institute of Health and Medical Research is the subject of the case study described in Section 4. We conclude in Section 5.

\subsection{Related Work}

Most works on the alignment measurement of EA viewpoint models highlight organization need of business - IS consistency enabling organization results improvement [6]. To satisfy this need, one solution is to compare its 'enterprise viewpoint' (including strategy) and its IS or IT viewpoints. From this alignment, heuristics have been defined, for instance, to provide warnings in case of misalignment [7]. Measurement methods concerning EA allow evaluation of this architecture in business terms (cost, benefit, risk impact analysis).

Other works about alignment measurement are based on strategic elements compared to operational elements [8]. Alignment measure is then based on quality criteria and measure ontology [9].

Significant works specific to IS model alignment with respect to organization strategy choose fitness measures [10]. These measures are based on alignment ratio. The quantity of business concepts well aligned with IS concepts is thus divided by the quantity of business concepts. Similar measures are proposed in [11] to estimate the alignment of an IS model with respect to a business model. This estimation concerns the 'computational viewpoint' with respect to the 'enterprise viewpoint', including the organization entities and their relationships.

Our previous work [12] about viewpoint models alignment proposes axiomatized measures based on such alignment ratios. Those ratios are between the quantity of well aligned elements from 'information viewpoint' with respect to 'enterprise viewpoint' and the quantity of 'information viewpoint' elements.

\section{EA Viewpoint Alignment Definition}

We propose to complete the EA viewpoint modeling process with an alignment measure, whatever the viewpoint. EA viewpoint modeling process consists in the design of one or more viewpoints that build upon the EA. 


\subsection{Alignment Definition Based on Systemic Approach}

In this paper, an alignment between two viewpoints is equivalent to the alignment between some models representing each aligned viewpoint [13]. Viewpoint models alignment needs concept mapping. We propose a systemic approach to define the models concepts. In the rest of the paper, a concept is so a unit or a relationship between units enabling their interaction.

The alignment is first defined for a $\mathrm{MM}_{-} \mathrm{V}_{\mathrm{W}}$ work viewpoint meta-model with respect to a $M M_{-} V_{R}$ reference viewpoint meta-model by:

$$
F_{A}: M M_{-} V_{W} \rightarrow M M_{-} V_{R}
$$

where one $M_{-} V_{W}$ concept (a MM_V $V_{W}$ unit or a $M M_{-} V_{W}$ unit relationship) is associated to a MM_ $V_{R}$ concept in the viewpoints meta-model.

\subsection{Viewpoint Models Units' Alignment Application}

We propose an alignment function, i.e. $\mathrm{f}_{\mathrm{A}}$, which conforms to $\mathrm{F}_{\mathrm{A}}$. The alignment objective is a mapping of a meta-model concept instance of a work viewpoint model with respect to meta-model concept instances of a reference viewpoint model. The units' alignment of the $M_{-} V_{W}$ work viewpoint model with respect to the $M_{-} V_{R}$ reference viewpoint model is defined by:

$$
\begin{aligned}
f_{A}: M_{-} V_{W} & \rightarrow \mathrm{P}\left(M_{-} V_{R}\right) \\
u_{W} & \mapsto\left\{u_{R i} ; u_{R i} \text { aligned with } u_{W}\right\}_{i \in[1 . . n]}, \text { where }
\end{aligned}
$$

- $\quad P\left(M_{-} V_{R}\right)$ is the power set of $M_{-} V_{R}$,

- $u_{w}$ is a $M_{-} V_{w}$ unit, $u_{R i}$ a $M_{-} V_{R}$ unit,

- $\mathrm{M}_{-} \mathrm{V}_{\mathrm{w}}$ conforms to $\mathrm{MM} \mathrm{V}_{\mathrm{w}}$ meta-model and $\mathrm{M}_{-} \mathrm{V}_{\mathrm{R}}$ conforms to $\mathrm{MM} \mathrm{V}_{\mathrm{R}}$ meta-model,

- $\mathrm{n}$ (that could be 0 ) is the quantity of the $\mathrm{M}_{-} \mathrm{V}_{\mathrm{R}}$ units aligned with $\mathrm{u}_{\mathrm{w}}$.

\subsection{Viewpoint Models Relationships' Alignment Application}

Alignment between unit relationships only takes into account work viewpoint units, which are aligned with at least one or more units of the reference viewpoint. We extend so the ' $\mathrm{f}_{\mathrm{A}}$ ' definition to the relationships of the viewpoint models:

$$
\begin{aligned}
f_{A}: M_{-} V_{W} & \rightarrow P\left(M_{-} V_{R}\right) \\
u r_{W} & \mapsto\left\{\begin{array}{l}
u r_{R i} ;\left(u r_{R i}=\left(u_{R i a}, u_{R i b}\right) \text { aligned with ur } r_{W}=\left(u_{W a}, u_{W b}\right)\right) \wedge \\
\left(u_{R i a} \in f_{A}\left(u_{W a}\right)\right) \wedge\left(u_{R i b} \in f_{A}\left(u_{W b}\right)\right)
\end{array}\right\}_{i \in[1 . . m]}, \text { where }
\end{aligned}
$$

- $\quad P\left(M_{-} V_{R}\right)$ is the power set of $M_{-} V_{R}$ reference model,

- $\mathrm{ur}_{\mathrm{w}}$ is a $\mathrm{M}_{-} \mathrm{V}_{\mathrm{w}}$ work model unit relationship between $\mathrm{u}_{\mathrm{wa}}$ and $\mathrm{u}_{\mathrm{wb}}$ units,

- $\mathrm{ur}_{\mathrm{Ri}}$ is a $\mathrm{M}_{-} \mathrm{V}_{\mathrm{R}}$ unit relationship and

- $m$ is the quantity of the $M_{-} V_{R}$ unit relationships aligned with $u_{w}$. 


\section{EA Viewpoint Alignment Measure}

Two measures of the alignment between viewpoint models are proposed. The first one enables to estimate the alignment of one concept of the work viewpoint model with respect to the reference viewpoint model. The second one provides an estimation of the alignment of the work viewpoint model with respect to the reference viewpoint model.

\subsection{Viewpoint Model Concept Alignment Measure}

A concept alignment measure is first proposed thanks to an axiomatization [14]. An axiom is an expected and understandable property of a concept alignment measurement. The following concept alignment (CA) axioms define the intuitive behavior of the alignment of one concept $s c_{W}$ of a work viewpoint model with respect to a reference viewpoint model. The first principle of the alignment behavior is that the alignment depends on the quantity of elements of the $f_{A}\left(s c_{W}\right)$ set. This quantity is represented by the function Card in this paper.

CA1 - One-to-one alignment. An alignment of one $\mathrm{sc}_{\mathrm{W}}$ concept (unit or relationship between units) of a $M_{-} V_{W}$ viewpoint model with respect to a $M_{-} V_{R}$ viewpoint model is perfect if the concept is aligned with only one concept of the $M_{-} V_{R}$ viewpoint model.

A perfect alignment enables to define without any ambiguity a concept of the work viewpoint model with respect to a concept of the reference viewpoint model.

CA2 - One-to-many alignment. An alignment of one $\mathrm{sc}_{\mathrm{W}}$ concept (unit or relationship between units) of a $M_{-} V_{W}$ viewpoint model with respect to a $M_{-} V_{R}$ viewpoint model is better if $\operatorname{Card}\left(\mathrm{f}_{\mathrm{A}}\left(\mathrm{sc}_{\mathrm{W}}\right)\right)$ decreases without reaching zero and is worse if $\operatorname{Card}\left(\mathrm{f}_{\mathrm{A}}\left(\mathrm{sc}_{\mathrm{W}}\right)\right)$ increases.

CA3 - One-to-zero alignment. An alignment of one $\mathrm{sc}_{\mathrm{W}}$ concept (unit or relationship between units, but not both) of a $M_{-} V_{W}$ viewpoint model with respect to a $M_{-} V_{R}$ viewpoint model is null if the concept is not aligned with any concept of $M_{-} V_{R}$.

The following concept weighted alignment (CWA) axiom defines the intuitive behavior of a weighted alignment.

CWA - Concept weighted alignment. A weighted alignment of a concept $\mathrm{sc}_{\mathrm{W}}$ (unit or relationship between units) of a $M_{-} V_{W}$ viewpoint model with respect to a $M_{-} V_{R}$ viewpoint model decreases if the weight of $\mathrm{sc}_{\mathrm{W}}$ decreases and increases if the weight of $\mathrm{sc}_{\mathrm{W}}$ increases.

CWA highlights that the alignment of a concept that is not significant for a viewpoint stakeholder of the organization must be investigated later than a prime one.

A $\mu_{\mathrm{CWA}}$ measure of the weighted alignment of the $\mathrm{sc}_{\mathrm{W}}$ concept such as $\mathrm{sc}_{\mathrm{W}} \in \mathrm{M}_{-} \mathrm{V}_{\mathrm{W}}$ with respect to a $M_{-} V_{R}$ viewpoint model could be the following: 


$$
\begin{aligned}
& \mu_{C W A}\left(s c_{W} / M_{-} V_{R}\right)=\omega_{W}\left(s c_{W}\right) \times\left(\frac{1}{\operatorname{Card}\left(f_{A}\left(s c_{W}\right)\right)}\right), \text { if } \operatorname{Card}\left(f_{A}\left(s c_{W}\right)\right) \neq 0 . \\
& \mu_{C W A}\left(s c_{W} / M_{-} V_{R}\right)=0, \text { if } \operatorname{Card}\left(f_{A}\left(s c_{W}\right)\right)=0
\end{aligned}
$$

where:

- $\quad \mathrm{M}_{-} \mathrm{V}_{\mathrm{R}}$ is the set of concepts (unit if $\mathrm{sc}_{\mathrm{w}}$ is a unit, relationship between units if $\mathrm{sc}_{\mathrm{w}}$ is a relationship between units) of the reference viewpoint model.

- $\quad \omega_{W}: M V_{W} \rightarrow[0,1]$ associates a weight to a concept of the $\mathrm{M}_{-} \mathrm{V}_{\mathrm{w}}$ model.

\subsection{Viewpoint Models Weighted Alignment Measure}

The alignment of a work viewpoint model with respect to a reference viewpoint model provides an estimation of the consistency between the two viewpoint models. The following axiom (GWA) defines the intuitive behavior of this weighted alignment deduced from the alignment axiomatization.

The principle of the weighted alignment behavior is that the weight of $\mathrm{sc}_{\mathrm{W}}$ and the weight of the $f_{A}\left(s_{W}\right)$ set constrain the alignment. This constraint means that the global weighted alignment estimation of each concept is balanced by these weights (weighted average).

GWA - Global Weighted Alignment. The weighted alignment between the concepts (units or relationships between units, but not both) of a $\mathrm{M}_{-} \mathrm{V}_{\mathrm{W}}$ work viewpoint model with respect to a $M_{-} V_{R}$ reference viewpoint model is estimated by the weighted average of all the non-weighted alignment estimation.

A $\mu_{\mathrm{GWA}}$ measure of the weighted alignment of $\mathrm{M}_{-} \mathrm{V}_{\mathrm{W}}$ with respect to $\mathrm{M}_{-} \mathrm{V}_{\mathrm{R}}$ consistent with (1) and with the GWA axiom could be:

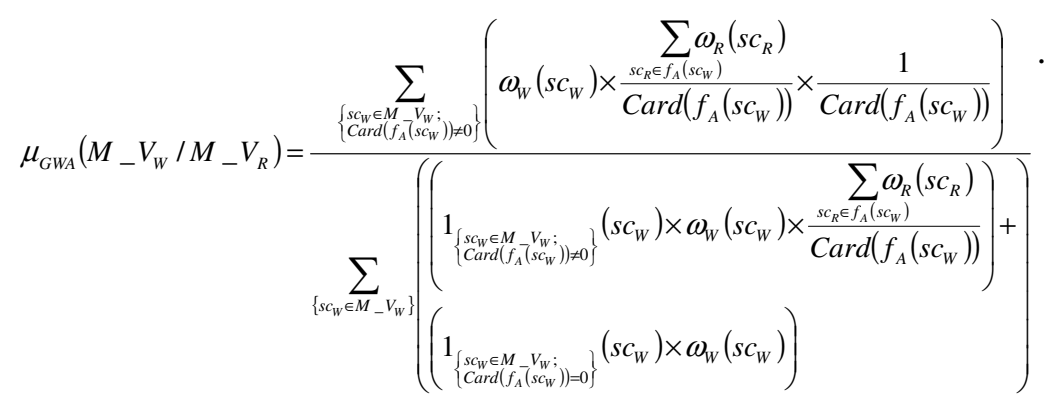

where:

- $\quad 1_{M_{-} V}$ is the indicator function defined on the M_V model concepts set;

- $\quad$ M_V is a set of model units nor a set of model relationships between units;

- $\omega_{W}: M_{-} V_{W} \rightarrow[0,1]$ associates a weight to a concept of the $\mathrm{M}_{-} \mathrm{V}_{\mathrm{w}}$ model;

- $\omega_{R}: M_{-} V_{R} \rightarrow[0,1]$ associates a weight to a concept of the $\mathrm{M}_{-} \mathrm{V}_{\mathrm{R}}$ model. 


\section{$4 \quad$ Real Case Study of Enterprise and Information Viewpoints Alignment}

We choose in this real case study the Reference Model for Open Distributed Processing (RM-ODP) framework [15]. The case study is the alignment of an 'information viewpoint' with respect to the 'enterprise viewpoint' that emphasizes the consistency of an IS with the supported organization's business. The IS takes place in an epidemiological research unit of INSERM, the French National Institute of Health and Medical Research [16]. The axioms proposed in the Section 3 are useful in this real study case because the vocabulary of the 'enterprise viewpoint' is different from the vocabulary of the 'information viewpoint'. Indeed, the epidemiology researchers use on the one hand a medical vocabulary in the 'enterprise viewpoint' and the statisticians or the data request designers use on the other hand a technical vocabulary in the 'information viewpoint'. A one-to-one alignment (see CA1 in the Subsection 3.1 ) is then useful to validate the association of every term of the technical vocabulary with one term of the medical vocabulary.

\subsection{Alignment Definition}

The alignment study case is restricted to meta-model units. For the reference 'enterprise viewpoint' $\left(\mathrm{V}_{\mathrm{R}}=\mathrm{V}_{\mathrm{E}}\right)$, the unit is the business activity, which composes a business process, as unit ( 24 activities compose 3 business processes dealing with the epidemiological studies).

The concepts chosen for the work 'information viewpoint' $\left(\mathrm{V}_{\mathrm{W}}=\mathrm{V}_{\mathrm{I}}\right)$ is the information block composed of functions that support a business activity ( 21 blocks designed in the epidemiological IS).

The alignment of the information blocks with respect to the business activities is justified by a relationship supports (specified inside the viewpoints meta-model of the INSERM) meaning that one information block may support one or more business activities.

\subsection{Information Block Weighted Alignment Measure}

The INSERM stakeholders have to apply first the units' alignment (see Subsection 2.2) of an information block with respect to the business activities. In this case study, the weight is deduced from a categorization of the information blocks. An information block category defined in [12] is 'Command' (for example, IBAccessRightManagement specific to one epidemiological system use) or 'Data' (for example, IBCatalogueVariableManagement transverse to more than one epidemiological system uses). The weight of an information block is 1.0 if 'Data' categorized or 0.5 if 'Command' categorized.

One alignment illustration is for example the IBCatalogueVariableManagement catalogue management information block that supports nine business activities: Provide data, Design the database, Map data, Filter data, Agree the exchange format, Give an access to the database, Check the database, Validate the scientific conformity, Validate the technical conformity. IBCatalogueVariableManagement is 
moreover categorized as a 'Data' information block $\quad \Rightarrow$ $\omega_{\mathrm{I}}(\{$ IBCatalogueVariableManagement $\left.\})=1.0\right)$,

$\Rightarrow \mu_{\mathrm{CWA}}\left(\{\right.$ IBCatalogueVariableManagement $\left.\} / \mathrm{V}_{\mathrm{E}}\right)=1.0 \times(1 / 9)=0.11(\mathrm{see}(\mathbf{1}))$.

The ranking has a beneficial effect during the alignment study of the 'information viewpoint' with respect to the 'enterprise viewpoint'. It enables indeed a prioritization of the information blocks for the alignment improvement activity.

\subsection{Information Viewpoint Weighted Alignment Measure}

For the global weighted alignment measure (see Subsection 3.2), the useful weights are on the one hand a weight associated to the concepts of the $V_{I}$ work 'information viewpoint' (see Subsection 4.2) and on the other hand a weight associated to the concepts of the $\mathrm{V}_{\mathrm{E}}$ reference 'enterprise viewpoint'.

In this case study, the weight associated to the concepts of the 'enterprise viewpoint' underlines that a business activity specific to the INSERM is more important than an activity external to the INSERM for the stakeholders.

The result of the estimation of the weighted alignment measure of the 'information viewpoint' units' alignment with respect to the 'enterprise viewpoint' is (see (2)):

$$
\mu_{G W A}\left(M_{-} V_{I} / M_{-} V_{E}\right)=0.47
$$

This weighted alignment measures of the 'information viewpoint' alignment with respect to the 'enterprise viewpoint' highlights the non-fulfillment of the business processes description by the INSERM.

\section{Conclusion}

The systemic approach enables to apply simply alignment between viewpoint models in relation with any EA framework. The alignment application between the unit relationships of the work viewpoint model and the unit relationships of the reference viewpoint model may be moreover automatically deduced from the units' alignment application.

Our real case study highlights nevertheless the complexity of the alignment behavior. The CWA concept weighted alignment axiom and the GWA global weighted alignment axiom introduce some knowledge specific to the viewpoint stakeholders. Relevant weights selected by the viewpoint stakeholders are one possibility enabling the improvement of the alignment estimation accuracy between viewpoint models. Decision aid could be an interesting approach to refine the weight of every viewpoint model element.

\section{References}

1. Zachman, J.A.: A Framework for Information Systems Architecture. IBM Systems Journal 26(3), 276-292 (1987)

2. Henderson, J.C., Venkatraman, N.: Strategic Alignment: Leveraging Information Technology for Transforming Organizations. IBM Systems Journal 32(1), 4-16 (1993) 
3. Chen, P.P.: Suggested Research Directions for a New Frontier - Active Conceptual Modeling. In: Embley, D.W., Olivé, A., Ram, S. (eds.) ER 2006. LNCS, vol. 4215, pp. 14. Springer, Heidelberg (2006)

4. IEEE Standards Association: IEEE Std 1471-2000 - IEEE Recommended Practice for Architectural Descriptions for Software-Intensive Systems-Description (2000), http: / /standards.ieee.org/reading/ieee/std_public/ description/se/1471-2000_desc.html

5. Barkmeyer, E.J., Feeney, A.B., Denno, P., Flater, D.W., Libes, D.E., Steeves, M.P., Wallace, E.K.: Concepts for Automating Systems Integration. National Institute of Standards and Technology (2003)

6. Sabherwal, R., Chan, Y.E.: Alignment between Business and IS Strategies: a Study of Prospectors, Analyzers and Defenders. Information Systems Research 12(1) (2001)

7. Pereira, C.M., Sousa, P.: Enterprise Architecture: Business and IT Alignment. In: ACM Symposium on Applied Computing, Santa Fe, New Mexico, USA (2005)

8. Thevenet, L.-H., Salinesi, C.: Aligning IS to Organization's Strategy: The InStAL Method. In: Krogstie, J., Opdahl, A.L., Sindre, G. (eds.) CAiSE 2007 and WES 2007. LNCS, vol. 4495, pp. 203-217. Springer, Heidelberg (2007)

9. Ruiz, F., Genero, M., García, F., Piattini, M., Calero, C.: A Proposal of a Software Measurement Ontology. In: Conference on Computer Science and Operational Research, Buenos Aires, Argentina (2003)

10. Etien, A., Rolland, C.: A Process for Generating Fitness Measures. In: Pastor, Ó., Falcão e Cunha, J. (eds.) CAiSE 2005. LNCS, vol. 3520, pp. 277-292. Springer, Heidelberg (2005)

11. Vasconcelos, A., Periera, C.M., Sousa, P., Tribolet, J.: Open Issues on Information System Architecture research domain: the Vision. In: International Conference on Enterprise Information System (2004)

12. Simonin, J., Bertin, E., Le Traon, Y., Jézéquel, J.-M., Crespi, N.: Analysis and Improvement of the Alignment between Business and Information System for Telecom Services. International Journal on Advances in Software 4(1\&2) (2011)

13. Lopes, D., Hammoudi, S., Abdelouahab, Z.: Schema matching in the context of model driven engineering: From theory to practice. In: Advances in Systems, Computing Sciences and Software Eng. Springer, Netherlands (2006)

14. Shepperd, M.J., Ince, D.: Derivation and Validation of Software Metrics. Oxford University Press (1993)

15. RM-ODP: ITU-T Recommendation X.901 to X.904 I ISO/IEC 10746-1 to 4 and X.911 । ISO/IEC 15414, http: / / www.rm-odp.net/

16. Goldberg, M., Quantin, C., Guéguen, A., Zins, M.: Bases de données médicoadministratives et épidémiologie: intérêts et limites. Courrier des Statistiques (124) (2008) (in French) 